\title{
Vlasov simulation of the microwave instability in space charge dominated coasting ion beams
}

\author{
O. Boine-Frankenheim and I. Hofmann \\ Gesellschaft für Schwerionenforschung (GSI), Planckstrasse 1, 64291 Darmstadt, Germany
}

(Received 16 May 2000; published 17 October 2000)

\begin{abstract}
Advanced storage ring concepts for intense ion beams often require operation far outside the stability boundaries provided by Landau damping. Whether a machine can be operated in such a regime depends on the phase space dilution after saturation of the microwave instability. A Vlasov simulation model is employed to analyze the saturation mechanisms in space charge dominated coasting beams. The stabilizing effect of space charge [I. Hofmann, Laser Part. Beams 3, 1 (1985)] is addressed to fluidlike mode coupling effects.
\end{abstract}

PACS numbers: 29.20.Dh, 29.27.Bd, 52.65.-y

\section{INTRODUCTION}

Intense beams in storage or accumulator rings operated below transition are an integral part of many advanced accelerator applications, such as, e.g., heavy ion driven inertial fusion (HIDIF) [1] and proton drivers for neutrino factories. The success of these applications depends crucially on the conservation of longitudinal beam quality during the required storage time. The longitudinal resistive microwave instability is regarded as one of the main limiting factors. Most of the theoretical as well as experimental work has been done mainly for highenergy machines operating at or above transition (see, e.g., [2]). It has been pointed out by several authors that below transition nonlinear space charge phenomena can be of fundamental importance. Based on the hydrodynamic equations for a cold beam, the relevance of nonlinear wave phenomena, such as wave steepening and solitary waves, was pointed out in Ref. [3]. Steepening of longwavelength modes (wavelengths much longer than the beam pipe circumference) observed in the heavy ion cooler experimental storage ring (ESR) was found in agreement with the cold fluid theory (see Ref. [4]). A kinetic simulation based on the Vlasov-Fokker-Planck equation was employed in Ref. [5] to analyze space charge induced longlived hole structures observed in the ESR experiment. The possibility of a nonlinear stabilization of the microwave instability in a space charge dominated beam was first studied by particle-in-cell (PIC) computer simulations in Ref. [6]. Using beam parameters relevant for a storage ring in a heavy ion fusion driver scenario, it was demonstrated that a sufficiently strong space charge impedance stabilizes the microwave instability. The stabilization was related to mode coupling and Landau damping provided by a self-generated thin tail of the velocity distribution. A detailed understanding of the stabilizing mechanisms and their range of validity in the impedance plane is crucial for high current ring design studies. In the present work we perform a detailed parameter study inside and outside the stability boundary. Our study is based on the direct numerical integration of the Vlasov equation ("Vlasov simulation"). For the purpose of resolving space charge induced long-time phenomena, the direct integration has the advantage of being "noiseless." Another advantage compared to a PIC code is the homogeneous resolution in phase space. Vlasov simulations are now routinely used in the analysis of nonlinear kinetic plasma phenomena (see, e.g., $[7,8]$ ).

The structure of the paper is as follows. In Sec. II we give the kinetic equation describing the time evolution in longitudinal phase space. In Sec. III we review the results of the linearized Vlasov theory for an intense beam. In Sec. IV we describe our Vlasov simulation model. A detailed discussion on the collective mechanisms leading to the damping of a finite perturbation in an intense ion beam is presented in Sec. V. In Sec. VI the Vlasov simulation code is applied to the microwave instability.

\section{KINETIC DESCRIPTION}

Let $\dot{\theta}_{0}=\omega_{0}$ be the angular frequency, $v_{0}$ the velocity of the synchronous particle, and $\dot{\theta}_{0}+\Delta \dot{\theta}$ and $v_{0}+v_{z}$ the angular frequency and velocity of a nonsynchronous particle in a ring of radius $R$. The coordinates in a system comoving with the synchronous particle are

$$
z=R \Delta \theta, \quad v_{z}=\dot{z}=R \Delta \dot{\theta} .
$$

The relative velocity $v_{z}$ is related to the momentum spread $\sigma=\Delta p / p_{0}$ by $v_{z}=-\eta \beta_{0} c \sigma$, with the frequency slip factor $\eta=1 / \gamma_{t}^{2}-1 / \gamma^{2}$, the relativistic factor $\gamma_{0}=1 /\left(1-\beta_{0}^{2}\right)^{1 / 2}$, and $\beta_{0}=v_{0} / c$. For the initial state we assume a coasting beam and a Maxwellian velocity distribution $f_{0}\left(v_{z}\right)$ with the initial FWHM momentum spread $\sigma_{0}$. The time evolution of the distribution $f\left(z, v_{z}, t\right)$ is governed by the Vlasov equation

$$
\frac{\partial f}{\partial t}+v_{z} \frac{\partial f}{\partial z}-\frac{q \eta}{\gamma_{0} m} E_{z} \frac{\partial f}{\partial v_{z}}=0
$$

with the total longitudinal electric field $E_{z}(z, t)$, the ion charge $q$, and the ion mass $m$. The line charge density $\rho_{L}$ and the rms momentum spread $\sigma$ are given through

$$
\rho_{L}(z, t)=q \int_{-\infty}^{\infty} f d v_{z}
$$




$$
\sigma^{2}=\frac{1}{\left(\eta v_{0}\right)^{2}} \int_{0}^{2 \pi R} \int_{-\infty}^{\infty} v_{z}^{2} f d v_{z} d z
$$

Fluctuations in the line density of a coasting beam cause a longitudinal electric field acting on the beam ions. In accelerator physics the concept of ring impedances is used to obtain the electric field amplitude resulting from a line density perturbation at the $n$th harmonic of the revolution frequency $\omega_{0}$,

$$
E_{n}=-\frac{1}{2 \pi R} Z_{n} I_{n}
$$

Here, $I_{n}=\beta_{0} c \rho_{L, n}$ and $E_{n}$ denote the amplitudes of the beam current and the resulting longitudinal electric field. $Z_{n}$ is the total ring impedance acting at the $n$th harmonic. At low beam energies the space charge impedance is usually dominant. For the case of a transversely uniform beam of radius $a$ in a circular beam pipe of radius $b$, it was shown in Ref. [3] that the longitudinal space charge impedance can be approximated as

$$
\frac{Z_{n}^{\mathrm{sc}}}{n}=-i \frac{g Z_{0}}{\beta_{0} \gamma_{0}^{2}} \frac{1}{1+\left(n / n_{c}\right)^{2}},
$$

with $Z_{0}=377 \Omega, g=1+2 \ln (b / a)$, and the cutoff harmonic $n_{c}=2 \pi R / \lambda_{c}$ determined by the length scale parameter $\lambda_{c}=\gamma_{0}^{-1} \pi a \sqrt{g}$. The space charge impedance is proportional to the harmonic number in the long wavelength regime and vanishes at wavelengths of the order of $\lambda_{c}$ due to the image charges on the inner side of the beam pipe.

In addition to the purely reactive space charge impedance [Eq. (6)], many of the vacuum chamber elements through which the beam travels have a cavitylike structure whose individual contributions to the total ring impedance can be represented as (shunt impedance $R_{s}$, quality factor $Q$ )

$$
Z_{n}^{\mathrm{cav}}=\frac{R_{s}}{1+i Q\left(\omega_{n} / \omega_{r}-\omega_{r} / \omega_{n}\right)},
$$

with the eigenfrequency $\omega_{r}$ and $\omega_{n}=n \omega_{0}$. Often the total impedance due to imperfections of the ring environment is described in terms of a broadband impedance $(Q=1)$ of $Z^{\mathrm{bb}} / n \lesssim 10 \Omega$ at resonance, with $\omega_{r}$ in the $\mathrm{GHz}$ range.

\section{REVIEW OF THE LINEARIZED THEORY}

The linear modes following from the linearization of the Vlasov equation are characterized by a complex frequency shift $\Delta \omega=\omega-n \omega_{0}$ (harmonic number $n$ ). The real part $\Delta \omega_{R}$ determines the coherent frequency shift and the imaginary part $\Delta \omega_{I}$ the damping or growth rate. It is convenient to introduce scaled impedances according to

$$
\begin{aligned}
V_{n}-i U_{n}= & \frac{2 I_{0} q}{\pi m c^{2} \beta_{0}^{2} \gamma_{0}|\eta| \sigma_{0}^{2}} \\
& \times\left[\operatorname{Re}\left(\frac{Z_{n}}{n}\right)+i \operatorname{Im}\left(\frac{Z_{n}}{n}\right)\right],
\end{aligned}
$$

with the average current $I_{0}$ and the ring impedance $Z_{n}$. For general operating points in $\left(U_{n}, V_{n}\right)$ space one evaluates the dispersion function for $\Delta \omega$ following from the linearized Vlasov equation. This determines the corresponding region of stability $\left(\Delta \omega_{I} \leq 0\right)$ that is provided by Landau damping. Figure 1 shows the stability boundary evaluated for a Maxwellian distribution function. We define $V_{\mathrm{bb}}$ as the scaled broadband impedance at resonance.

For the beams inside the HIDIF storage rings, for example, one typically assumes that their longitudinal properties are dominated by the space charge impedance. In the limit of a space charge dominated beam $\left(U_{n} \gg 1\right.$ and $U_{n} \gg V_{n}$ ), we can use the dispersion relation for a cold beam to obtain the coherent frequency shift explicitly as

$$
\frac{\Delta \omega_{R}}{n S}=U_{n}^{1 / 2},
$$

with the incoherent frequency shift $S=-\eta \omega_{0} \sigma / 2$. By using the space charge impedance Eq. (6) in the long wavelength approximation we define the space charge parameter,

$$
U_{\mathrm{sc}}=U_{n \ll n_{c}}=\frac{2 I_{0} q g Z_{0}}{\pi m c^{2} \beta_{0}^{3} \gamma_{0}^{3}|\eta| \sigma_{0}^{2}} .
$$

The phase velocity of long-wavelength modes is $c_{0}=$ $R S U_{\mathrm{sc}}^{1 / 2}$. A small initial current perturbation can be represented as a superposition of slow $\left(c_{0}<0\right)$ and fast $\left(c_{0}>\right.$ $0)$ modes. The space charge parameter $U_{\mathrm{sc}}$, defined in Eq. (10), is a measure of the magnitude of the coherent velocity $c_{0}$ relative to the incoherent thermal velocity of the beam ions $R S$. As the space charge impedance approaches

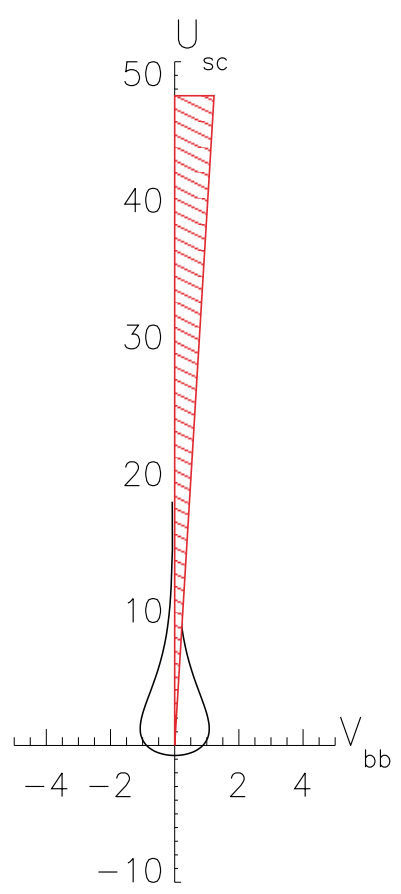

FIG. 1. (Color) The stability boundary provided by linear Landau damping (solid black line) together with the area (red) of "stabilization due to space charge induced mode coupling." 
wavelengths around $\lambda_{c}$, the phase velocity of these modes $c_{n}=R S U_{n}^{1 / 2}$ decreases.

For the imaginary part of the frequency shift in the limit of a space charge dominated beam we obtain

$$
\frac{\Delta \omega_{I}}{n S}= \pm \frac{1}{2} \frac{V_{n}}{U_{n}^{1 / 2}}+\frac{\pi}{2} U_{n}^{3 / 2}(R S)^{2}\left(\frac{d f_{0}}{d v_{z}}\right)_{v_{z}=c_{n}} .
$$

The first term on the right-hand side (rhs) of Eq. (11) follows from the dispersion relation for a cold beam and leads to the growth of slow modes and the damping of the fast modes due to a resistive impedance $\left(V_{n}>0\right)$. The second term on the rhs of Eq. (11) accounts for Landau damping due to resonant ions with $v_{z}= \pm c_{n}$ in the limit of weak damping $\left(\Delta \omega_{I} \ll \Delta \omega_{R}\right)$. Landau damping causes the damping of small amplitude modes provided that $d f_{0} / d v_{z}<0$.

\section{SIMULATION MODEL}

In order to analyze the nonlinear mechanisms leading to the saturation of the microwave instability we have to integrate the Vlasov equation [Eq. (2)], together with the self-consistent electric fields, numerically. The integration (Vlasov simulation) is performed on a grid in $\left(z, v_{z}\right)$ space using the time splitting scheme described in Ref. [9] and more recently in Ref. [10]. At each time step the electric space charge field is calculated from a Poisson solver on a grid in $(r, z)$ space, assuming a transversely uniform beam of radius $a\left[\rho(r, z)=\rho_{L}(z) /\left(\pi a^{2}\right)\right]$ in a conducting beam pipe of radius $b$. The longitudinal field is found from averaging the $r$ dependence over the homogeneous distribution function. Other possible contributions to the total electric field are calculated each time step using their impedance representation. The relevant numerical parameters are the number of points in $z$ and $v_{z}$ (respectively, $N_{z}$ and $N_{v}$ ), the cutoff velocity $v_{\max }$, and the time step $\Delta t$. The simulation of long-time phenomena in space charge dominated beams is complicated by the smallness of the cutoff wavelength $\lambda_{c}$ relative to typical ring circumferences. For the grid spacing along the $z$ axis we have to require $\Delta z<\lambda_{c}$ also to prevent numerical instabilities. In our simulations we choose a beam pipe with $b=0.05 \mathrm{~m}$ and $a / b=0.5$ resulting in $\lambda_{c}=0.12 \mathrm{~m}$. To limit the number of points along the $z$ axis to $N_{z}=512$ we restrict our numerical study to a periodic beam pipe of the length $L=5 \mathrm{~m}$ leading to a cutoff harmonic $n_{c} \approx 41$ in our simulation model. The mesh in velocity space must be dense enough to resolve the initial Maxwellian velocity distribution (FWHM width $2 R S$ ) and large enough for the coherent velocity $c_{0} \gg R S$. We typically chose the cutoff velocity at $v_{\max } \approx 4 c_{0}$ and the number of points as $N_{v}=400$. Actually, the choice of $N_{v}$ is more difficult if we are interested in the long-time evolution of nonlinear phenomena involving small electric fields, such as, e.g., nonlinear Landau damping. In this case the particle motion is close to free streaming. If the grid spacing is $\Delta v$ and $n$ is the initially exited mode number, there is a recurrence occurring at $T_{R}=2 \pi R /(n \Delta v)$, that can be easily detected when simulating linear Landau damping (see also [7]). The recurrence time should always exceed the simulation time $T$. Taking $n=n_{c}$ we obtain $T_{R}=\lambda_{c} / \Delta v$. Finally, the time step is determined through $\Delta t<\Delta z / v_{\max }$.

\section{NONLINEAR COLLECTIVE PHENOMENA IN STABLE BEAMS}

In this section we ignore any resistive impedance $\left(V_{n}=\right.$ 0 ) and account for the effect of space charge only. By analyzing the damping of an initial current modulation $\delta I$ of the form

$I(z, t)=I_{0}+\delta I=I_{0}+I_{n} \cos \left(n z / R-\Delta \omega_{R} t\right)$

we can isolate the mechanisms relevant for the saturation of the longitudinal instability in space charge dominated beams. The knowledge of the damping rate in the presence of space charge is important for the prediction of instability thresholds and saturation levels.

\section{A. Nonlinear Landau damping}

Landau damping is commonly regarded as the major mechanism responsible for the damping of coherent modes and thus for beam stability. The concept of Landau damping is the assumed basis of "overshoot" theories (see, e.g., Refs. [11]) formulated in the framework of quasilinear theory. Quasilinear theory predicts the saturation level of an unstable mode, assuming that stability is reestablished by Landau damping due to formation of a quasilinear plateau (see, e.g., [12]). However, from the nonlinear theory of electron plasma waves it is well known that the concept of Landau damping fails for sufficiently large initial wave amplitudes ("nonlinear Landau damping"). This was confirmed in Ref. [7] by means of Vlasov simulations. Experimental results on nonlinear Landau damping in a high-energy proton beam were reported in Ref. [13].

Here we analyze the validity of Landau damping for a beam current modulation [Eq. (12)] at a harmonic $n$ close to the cutoff harmonic (microwave regime). The breakdown of Landau damping is due to beam ions trapped in the self-excited space charge field of amplitude $E_{n}=$ $I_{n}\left|Z^{\mathrm{sc}}\right| /(2 \pi R)$. Ions trapped in the space charge field perform synchrotron oscillation with the so called bounce frequency

$$
\frac{\Delta \omega_{B}}{n S}=\left(\frac{I_{n}}{I_{0}}\right)^{1 / 2} \frac{\Delta \omega_{R}}{n S}=\left(\frac{I_{n}}{I_{0}}\right)^{1 / 2} U_{n}^{1 / 2} .
$$

The concept of Landau damping fails if the damping time exceeds the trapping time. In terms of the frequency shifts the range of validity is $\Delta \omega_{B} \ll \Delta \omega_{I}$. The condition $\Delta \omega_{B}=\Delta \omega_{I}$ determines a "threshold" current perturbation 


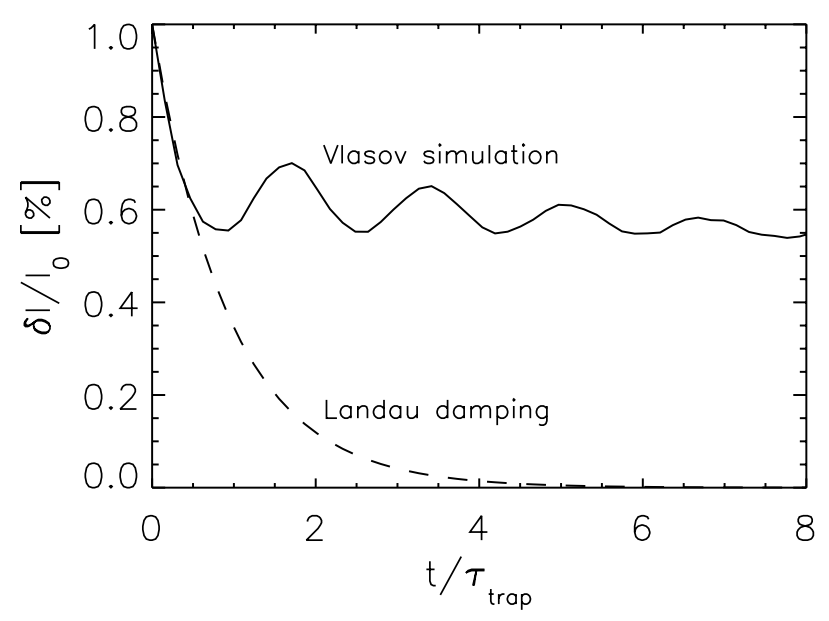

FIG. 2. Time evolution of the current amplitude obtained from the Vlasov simulation in comparison with Landau damping [Eq. (11)].

$$
\delta I_{\mathrm{th}}=I_{0}\left(\frac{\Delta \omega_{I}}{\Delta \omega_{R}}\right)^{2}
$$

For initial perturbations exceeding $\delta I_{\text {th }}$, one can expect the breakdown of the linearized Vlasov theory and thus of the concept of Landau damping. This is demonstrated using our Vlasov simulation model for a space charge parameter $U_{\mathrm{sc}}=10$ and an initial modulation at $n=12$ (corresponding to a wavelength of $0.42 \mathrm{~m}$ ) with $\delta I / I_{0}=$ 0.01 , exceeding $\delta I_{\mathrm{th}} / I_{0}$ by a factor of 10 . Figure 2 shows the time evolution of the current amplitude obtained from the simulation. For $t \approx 0.5 \tau_{\text {trap }}\left(\tau_{\text {trap }}=\Delta \omega_{B}^{-1}\right)$, the current amplitude decreases exponentially with the Landau damping rate

$$
\begin{aligned}
\tau_{L}^{-1} & =\left.\frac{\pi}{2} U_{n}^{3 / 2}(R S)^{2} \frac{d f_{0}}{d v_{z}}\right|_{v_{z}=c_{n}} \\
& =-\pi U_{n}^{2}(\ln 2)^{3 / 2} \exp \left(-U_{n} \ln 2\right)
\end{aligned}
$$

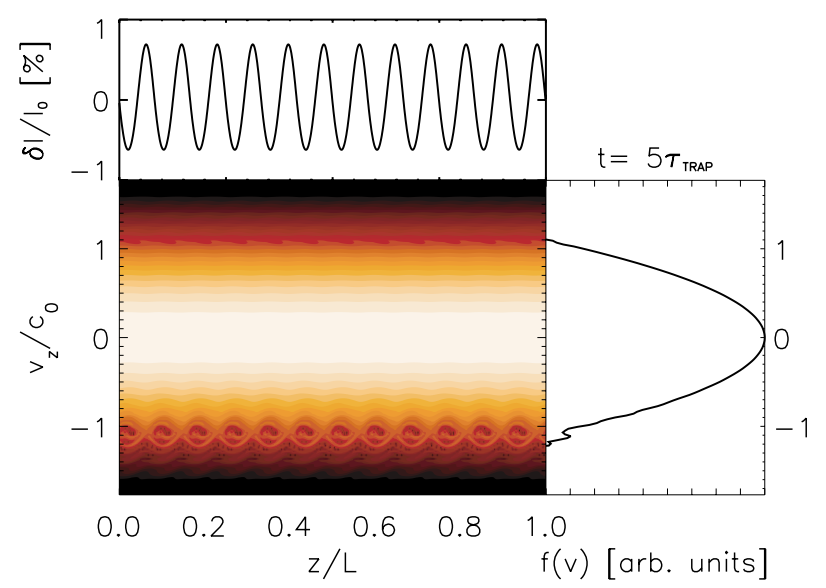

FIG. 3. (Color) Contour plot of the asymptotic distribution function $\ln f\left(z, v_{z}\right)$ together with the corresponding line density $\rho_{L}(z)$ and the velocity distribution $\ln f\left(v_{z}\right)$ obtained from the Vlasov simulation by starting with a current modulation at $n=12$ and $U_{\mathrm{sc}}=10$. following from Eq. (11) with $V_{n}=0$. For times exceeding $\tau_{\text {trap }}$ we observe amplitude oscillations with the bounce frequency. The asymptotic state shown in Fig. 3 is characterized by a strong local deviation of the velocity distribution from the initial Maxwellian (around $v_{z} \approx$ $-1.2 c_{0}$ ) and by a persistent current amplitude determined by the ions trapped in the self-consistent space charge field. Such a state is commonly called a Bernstein-GreenKruskal mode [14] in plasma physics.

Summarizing the discussion on nonlinear Landau damping we can say that in space charge dominated beams even small perturbations of the order of a few percent do not damp to zero, although resonant ions are present. Therefore, the concept of Landau damping (e.g., due to the formation of a quasilinear plateau) is at least questionable in order to predict the saturation amplitudes of unstable modes.

\section{B. Wave steepening and breaking}

So far we assumed that mode coupling is irrelevant and focused on the time evolution at an isolated harmonic. For sufficiently large amplitudes this assumption fails due to the broadband nature of the space charge impedance [Eq. (6)], leading to a coupling of all harmonics up to $n \approx n_{c}$. The most simple manifestation of mode coupling towards higher harmonics is represented by the phenomenon of wave steepening that can be described in the cold fluid limit $\left(\sigma_{0}=0\right)$ of the Vlasov equation. If we use the space charge electric field resulting from a general line density modulation in the long wavelength approximation

$$
E_{z}^{\mathrm{sc}}=-\frac{g}{4 \pi \epsilon_{0} \gamma_{0}^{2}} \frac{\partial \rho_{L}}{\partial z},
$$

the equations for the evolution of the line density and the fluid velocity $\left(u=\left\langle v_{z}\right\rangle\right)$ are

$$
\frac{\partial \rho_{L}}{\partial t}+\frac{\partial}{\partial z}\left(\rho_{L} u\right)=0, \quad \frac{\partial u}{\partial t}+u \frac{\partial u}{\partial z}=-\frac{c_{0}^{2}}{\rho_{L 0}} \frac{\partial \rho_{L}}{\partial z},
$$

with the average line density $\rho_{L 0}$ and the phase velocity $c_{0}=R S U_{\mathrm{sc}}^{1 / 2}$. By choosing appropriate initial conditions we can either excite a backward (slow) $c_{0}<0$ or a forward (fast) $c_{0}>0$ running wave. The analytic solution of the nonlinear cold fluid equations (see Ref. [4]) shows that an initial harmonic line density perturbation develops into a nonlinear wave with a steep gradient, causing the generation of higher order harmonics, that is, of shorter wavelength harmonics. The time instant $\tau_{\text {break }}$ at which $\partial \rho_{L} / \partial z$ becomes infinite ("wave breaking") is given by

$$
\tau_{\text {break }}^{-1}=\frac{3}{2} \frac{I_{n}}{I_{0}} \Delta \omega_{R}
$$

In Ref. [4] it was shown that the cold fluid model describes very well the wave steepening observed in ESR experiments for $t<\tau_{\text {break }}$. The time evolution for $t \gtrsim$ $\tau_{\text {break }}$ cannot be obtained by means of Eqs. (17) because 
of the long wavelength approximation for the space charge field. Nevertheless, one can regard $\tau_{\text {break }}$ as the time scale for mode coupling towards higher order modes. If linear Landau damping is suppressed by particle trapping $\left(\tau_{L}>\tau_{\text {trap }}\right)$, mode coupling due to wave steepening becomes the dominant decay mechanism ("decay rate" $\tau_{\text {break }}$ ) for an initial harmonic perturbation with $n \ll n_{c}$.

\section{Solitons}

The nonlinearity in the fluid equations [Eqs. (17)] leads to wave steepening. On the other hand, if the phase velocity depends strongly on wavelength (dispersion), steep gradients will tend to smooth out. A solitary wave results when the nonlinear steepening is balanced by the dispersive spreading, yielding a localized disturbance which propagates without distortion. Solitary waves of different heights propagate with different velocities $c_{s}$. The term soliton describes solitary waves which maintain their identity and shape after collisions with other solitary waves traveling at different speeds. Based on the cold fluid equations including dispersion the possibility of solitary waves in particle beams was pointed out in Ref. [3]. Dispersion is introduced by the shielding provided by the beam pipe, causing the phase velocity to drop according to

$$
c_{n}=\frac{c_{0}}{\sqrt{1+\left(n / n_{c}\right)^{2}}} .
$$

By comparing Eq. (19) with the phase velocity of ionacoustic waves in plasmas (see, e.g., [12]) we see that both expressions are exactly the same if we exchange the Debye length $\lambda_{D}$ by $k_{c}^{-1}=R / n_{c}$. In fact, there is an analogy between the shielding by the beam pipe and the Debye shielding of ion-acoustic waves in plasmas, where soliton solutions exist. For weak dispersion ion-acoustic solitons are governed by a Korteweg-de Vries (KdV) equation (see, e.g., [12]). By using the analogy with ion-acoustic waves, exchanging $k_{D}$ by $k_{c}$, we obtain the stationary solution of the KdV equation for a particle beam

$$
\frac{\delta \rho_{L}}{\rho_{L 0}}=3 \delta M \operatorname{sech}^{2}\left[(\delta M / 2)^{1 / 2} k_{c}\left(z-M c_{0} t\right)\right]
$$

with $\delta M=M-1$ (Mach number $M=c_{s} / c_{0}$ ). A more rigorous treatment would start from the standard small parameter expansion $(\delta M \ll 1)$ of the cold fluid equations [Eqs. (17)] in the limit of weak dispersion [correction $\sim \partial^{3} \rho_{L} / \partial z^{3}$ to Eq. (16)].

The question of whether or not solitons can be generated from an arbitrary current perturbation cannot be answered easily. One condition is that the perturbation must be sufficiently strong to provide the energy for the creation of a soliton. Kinetic effects that lie beyond the cold fluid approximation can cause the decay of solitons.

We use the Vlasov simulation to see whether solitons can be generated from an initial current modulation.
Unlike our treatment of Landau damping in Sec. V A, we start from a long-wavelength modulation at the lowest harmonic $(n=1)$ of our simulation model, corresponding to a wavelength of $5 \mathrm{~m}$. The space charge parameter is $U_{\mathrm{sc}}=10$, and the initial amplitude is $\delta I / I_{0}=0.1$. Figure 4 shows the time evolution of the distribution function together with the velocity distribution and the line density. At $t \approx \tau_{\text {break }}$ we clearly observe the steepening of the wave. Subsequently, a train of localized structures develops on the crest of the wave. The localized structures are of the $\operatorname{sech}^{2}$ type. Their width is of the order of several cutoff wavelengths, but they are not stationary. Later in time ( $\left.t=18 \tau_{\text {break }}\right)$ we observe (see Fig. 5) the formation of a single solitonlike structure of the form Eq. (20). Also, this structure finally decays, probably due to the exchange of energy with reflected ions, but similar structures keep on appearing and disappearing. Exchange of energy decelerates a small fraction of the beam ions towards $v_{z} \approx-2 c_{0}$.

It is worth noting that line density profiles similar to Fig. 4 were observed in a cooled $\mathrm{Ca}^{20+}$ beam in the ESR interacting with the rf cavity impedance tuned to the second harmonic, corresponding to a wavelength of $55 \mathrm{~m}$. In Ref. [15] the observed train of localized structures on the crest of the steepened wave was related to possible higher order resistive impedances in the ESR. Our work shows that these structures can develop due to space charge only.
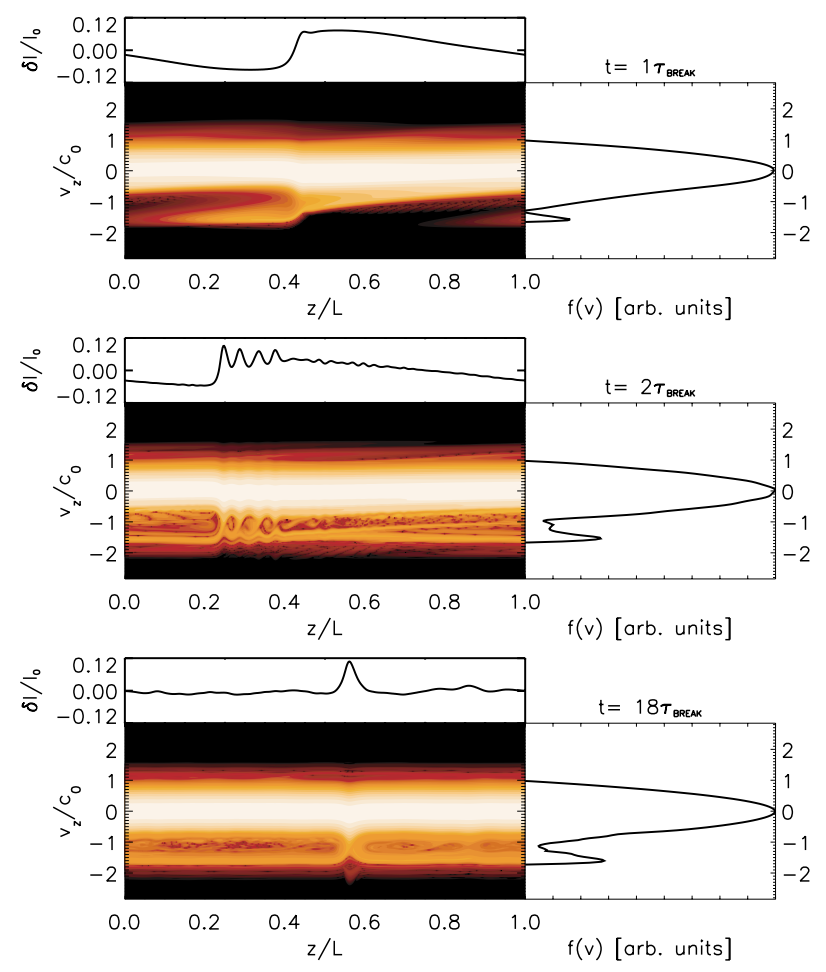

FIG. 4. (Color) Contour plots of the distribution function $\ln f\left(z, v_{z}\right)$ together with the corresponding line density $\rho_{L}$ and the velocity distribution $\ln f\left(v_{z}\right)$ obtained from the Vlasov simulation by starting with a current modulation at $n=1$ and $U_{\text {sc }}=10$. 


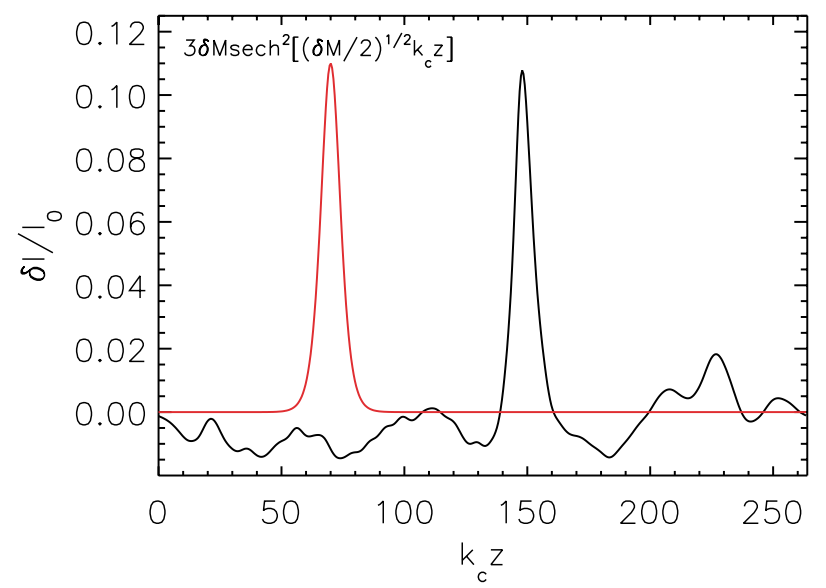

FIG. 5. (Color) Line density obtained from the Vlasov simulation at $t=18 \tau_{\text {break }}$ (black line) and Eq. (20) with $\delta M=0.037$ (red line).

\section{STABILIZATION OF THE MICROWAVE INSTABILITY}

We simulate the microwave instability in a space charge dominated beam $\left(U_{\mathrm{sc}}=10\right)$ caused by a broadband impedance [Eq. (7)] with $Q=1$ centered at $n_{r}=12$, corresponding to a wavelength of $42 \mathrm{~cm}$. Figure 6 shows the time evolution of the current amplitudes for $V_{\mathrm{bb}}=1$. The exponential growth of the amplitude of the $n=12$ mode is initiated by a harmonic line density modulation of $0.5 \%$. Relative to the exponential growth predicted by linear theory (instability growth rate $\tau_{I}^{-1}$ ) the growth slows down at $t \approx 3 \tau_{I}$ and $I_{n} / I_{0} \approx 5 \%$ as the mode turns nonlinear and coupling towards higher harmonics becomes effective. The dominating current amplitude $\left(n=n_{r}\right)$ reaches a peak value of $I_{n} / I_{0} \approx 40 \%$ at $t \approx 7 \tau_{I}$ before it decreases and finally vanishes as the bulk velocity distribution shifts towards $v_{z} \approx-c_{0}$ (see Fig. 7). The saturation of the instability leads to a broadening ("heating") and to a shift (deceleration) of

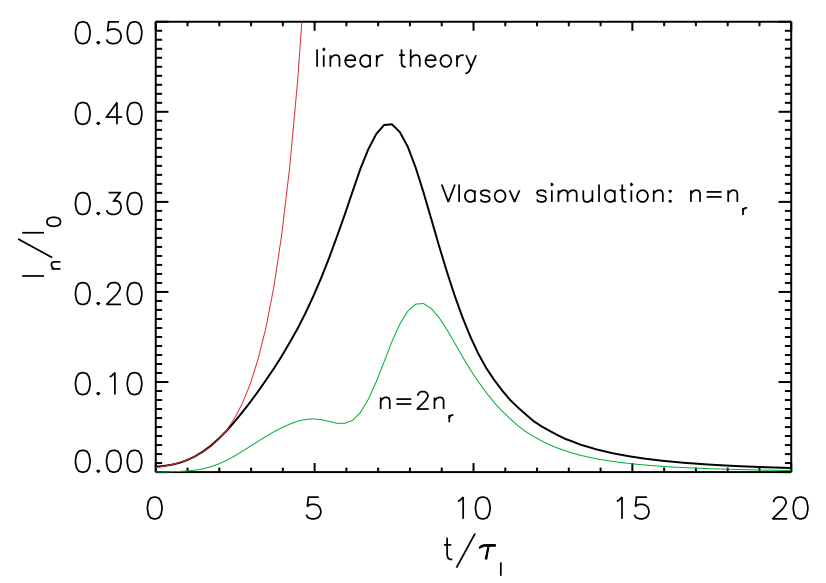

FIG. 6. (Color) Time evolution of the current amplitudes obtained from the Vlasov simulation for $U_{\mathrm{sc}}=10, V_{\mathrm{bb}}=1$, and $n_{r}=12$. the velocity distribution relative to the initial distribution. Because of the observation (Fig. 6) that mode coupling is relatively weak, the relevant saturation mechanism is the trapping of ions in the potential created by the growing current perturbation. Ion trapping causes formation of hole structures in phase space and corresponding localized line density dilutions that can both be seen in Fig. 7 $\left(t=7 \tau_{I}\right)$. Similar trapped particle hole structures were also observed in Ref. [5] during the saturation of the longitudinal instability in the long-wavelength regime.

The destructive effect of the microwave instability on the beams distribution function is strongly reduced if the resistive impedance is lowered below $V_{\mathrm{bb}} \approx 0.15$. Figure 8 shows the evolution of the current amplitudes for $V_{\mathrm{bb}}=0.1$. Again, the exponential growth predicted by linear theory for the $n=12$ mode starts to overestimate the current amplitude at $t \approx 3 \tau_{I}$ and $I_{n} / I_{0} \approx 5 \%$ as coupling towards higher harmonics becomes effective. The growth stops at $I_{n} / I_{0} \approx 11 \%$ and $t \approx 5 \tau_{I}$ due to the generation of subharmonics, such as $n_{r} / 2$ and $n_{r} / 3$, and a corresponding sudden reduction in the number of line density peaks (see Fig. 9). The localized line density peaks coalesce into a single solitonlike structure. Later in time the localized structure decays, but similar structures keep on appearing and disappearing. The energy supplied by the instability at $n=n_{r}$ is transformed via space charge induced mode coupling into a persistent amount of coherent energy and into a relatively small amount of
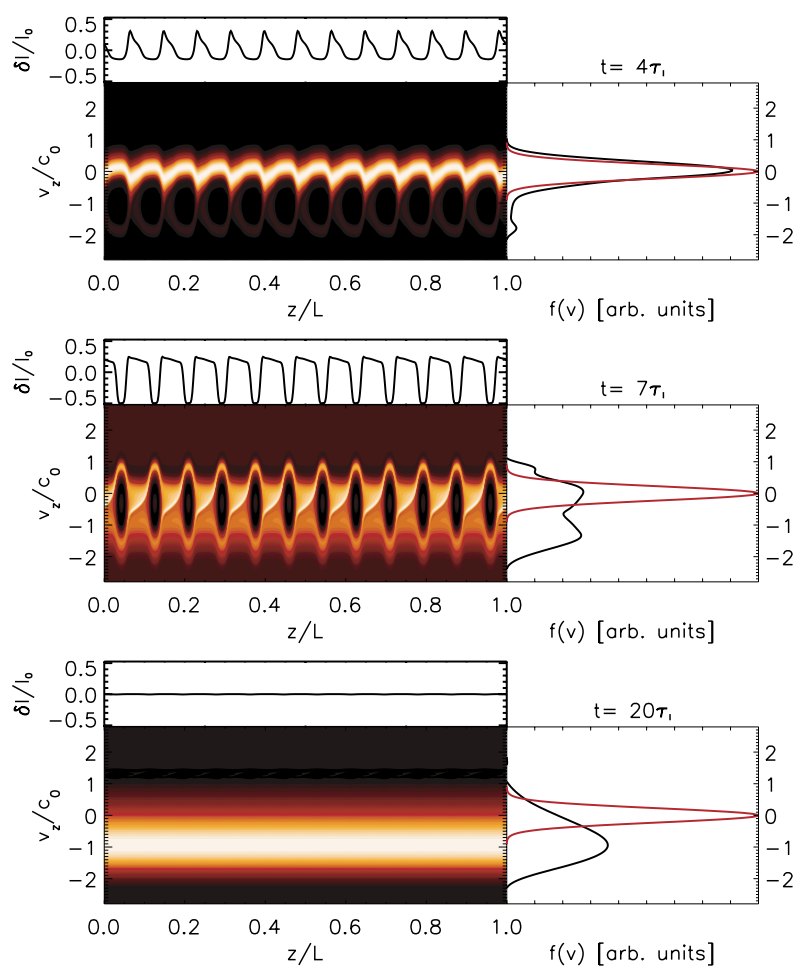

FIG. 7. (Color) Contour plots of the distribution function $f\left(z, v_{z}\right)$ together with the line density $\rho_{L}(z)$ and the velocity distribution $f\left(v_{z}\right)$ obtained from the Vlasov simulation for $U_{\mathrm{sc}}=10, V_{\mathrm{bb}}=1$, and $n_{r}=12$. 


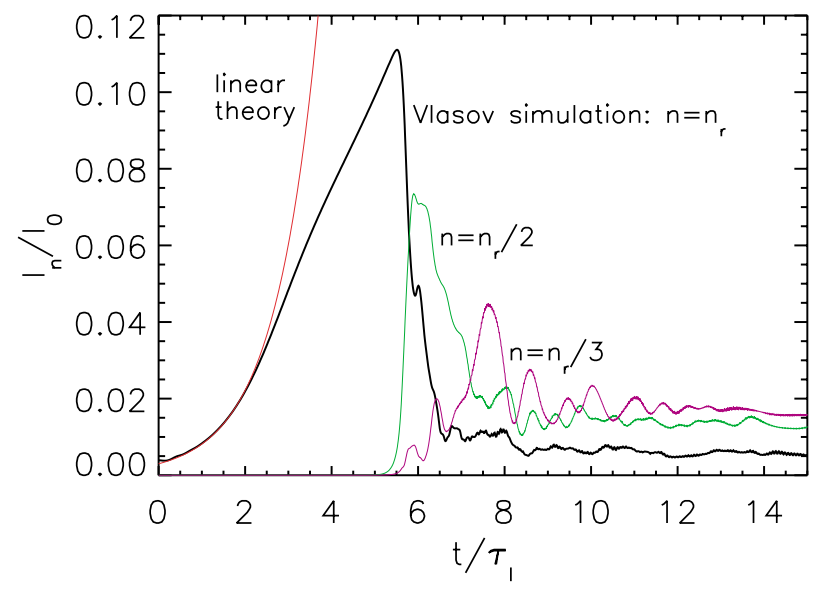

FIG. 8. (Color) Time evolution of the current amplitudes obtained from the Vlasov simulation for $U_{\mathrm{sc}}=10, V_{\mathrm{bb}}=0.1$, and $n_{r}=24$.

decelerated ions forming a bump around $v_{z} \approx-2 c_{0}$. To make sure that the instability saturation is not affected by the reduced length of our simulation model or by the grid spacing, we performed various test runs using larger $L$ and smaller grid spacing. Figure 10 shows, e.g., that the observed phenomena are the same for $L=10 \mathrm{~m}$.

Below $V_{\mathrm{bb}} \approx 0.15$ the destructive effect of the instability is strongly reduced, therefore the description "space charge induced stabilization" seems appropriate. Below
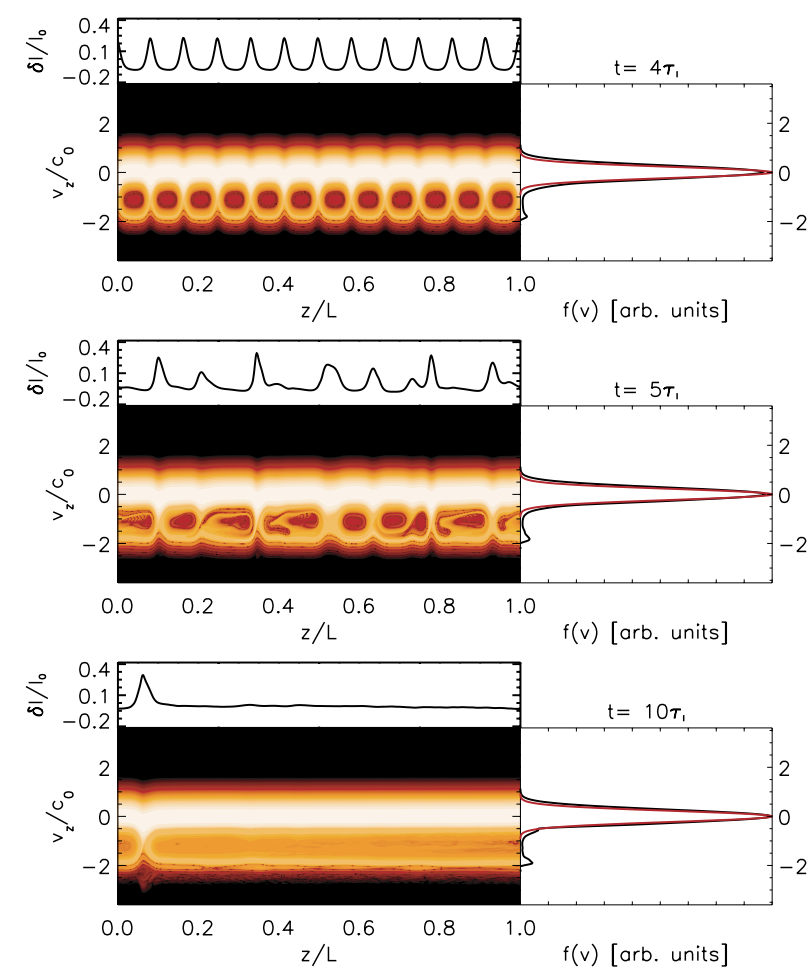

FIG. 9. (Color) Contour plots of the distribution function $\ln f\left(z, v_{z}\right)$ together with the line density $\rho_{L}(z)$ and the velocity distribution $f\left(v_{z}\right)$ obtained from the Vlasov simulation for $U_{\mathrm{sc}}=10, V_{\mathrm{bb}}=0.1$, and $n_{r}=12$.
$V_{\mathrm{bb}} \approx 0.15$ we still observe exponential growth, but the peak current amplitude at $n=n_{r}$ is effectively limited by space charge induced mode coupling, thereby inhibiting the phase space "blowup." The shape of the final velocity distribution differs from the initial distribution only because of a bump around $v_{z} \approx-2 c_{0}$. So far we have not been successful in providing an analytical explanation for the sudden decay into subharmonics observed in the simulation. Unlike the simple manifestation of mode coupling towards higher harmonics through wave steepening (Sec. V B), an accurate explanation of the observed coupling towards subharmonics and the final generation of a single localized structure is more complex. Analytic as well as experimental work on parametric three-wave coupling and solitary wave generation in high energy machines was presented in Refs. [13,16]. The extension of this work to low energy, space charge dominated beams is presently under investigation and will be the topic of future publications.

By tuning the eigenfrequency of the cavity to different harmonics of our Vlasov simulation model,we study the saturation mechanisms for $1 \leq n_{r} \leq 36$. For $U_{\mathrm{sc}}=10$ and $V_{\mathrm{bb}} \lesssim 0.15$, we find that the sudden decay towards subharmonics is the dominant saturation mechanism for $6 \lesssim n_{r} \lesssim 36$, corresponding to wavelengths of the order of $1 \mathrm{~m}$ down to $10 \mathrm{~cm}$. Below $n_{r} \approx 6$ ("long-wavelength regime"), wave steepening as shown in Fig. 4 becomes the
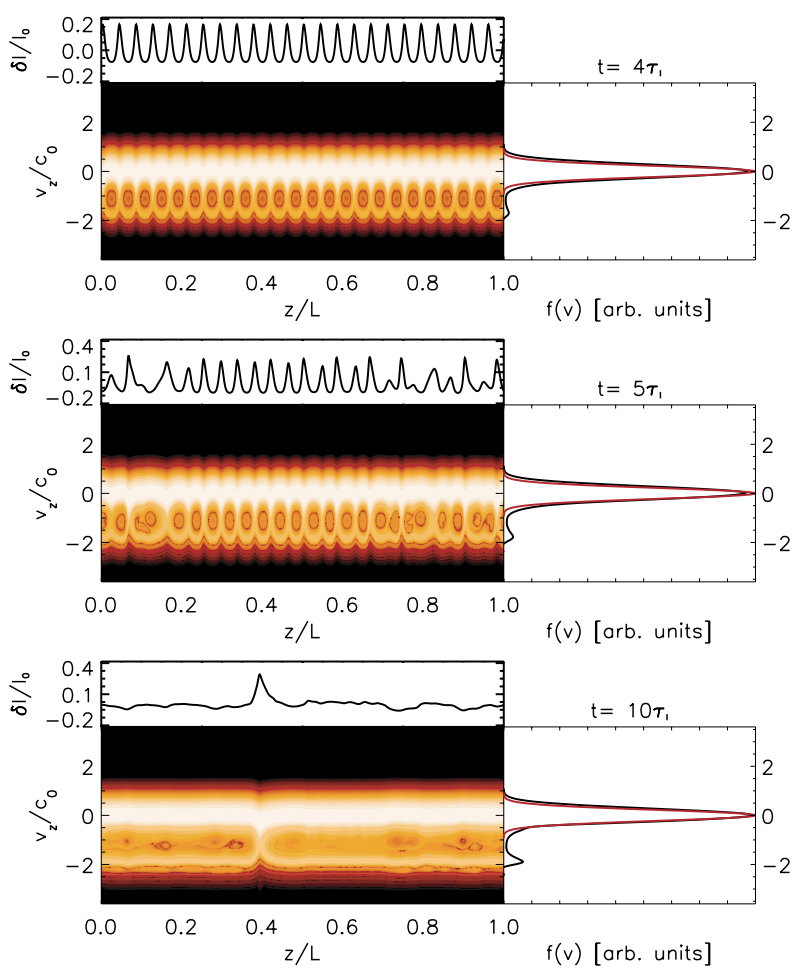

FIG. 10. (Color) Contour plots of the distribution function $\ln f\left(z, v_{z}\right)$ together with the line density $\rho_{L}(z)$ and the velocity distribution $f\left(v_{z}\right)$ obtained from the Vlasov simulation (with $L=10 \mathrm{~m}$ ) for $U_{\mathrm{sc}}=10, V_{\mathrm{bb}}=0.1$, and $n_{r}=24$. 
dominant saturation mechanism. For $n_{r} \gtrsim n_{c}$ and initial perturbations of the order of $1 \%$, we find that modes are stabilized by Landau damping for $V_{\mathrm{bb}} \lesssim 0.5$.

From our simulation we find that, for a given $n_{r} \lesssim$ $n_{c}$ and $U_{\mathrm{sc}} \gg 1$, the "stability boundary" provided by space charge induced mode coupling scales according to $\sim U_{\mathrm{sc}} / V_{\mathrm{bb}}$ (or equivalently $\sim Z^{\mathrm{sc}} / Z^{\mathrm{bb}}$ ), meaning that for increased $U_{\mathrm{sc}}$ a higher $V_{\mathrm{bb}}$ can be tolerated. In the linear theory (Sec. III), the stability boundary is determined by equating the instability rise time $\tau_{I} \sim U_{\mathrm{sc}}^{1 / 2} / V_{\mathrm{bb}}$ with the Landau damping time $\tau_{L}$ [see Eq. (9)]. For space charge dominated beams, the Landau damping time must be replaced by a characteristic time scale for mode coupling like the wave breaking time $\tau_{\text {break }} \sim U_{\text {sc }}^{-1 / 2}$ [see Eq. (18)]. The resulting stability boundary, determined by $\tau_{I}=\tau_{\text {break }}$, shows the $U_{\mathrm{sc}} / V_{\mathrm{bb}}$ scaling obtained in the simulations. Figure 1 shows a simplified sketch of the space charge stabilized region found within our simulation model for the microwave instability.

\section{CONCLUSIONS}

By means of Vlasov simulations we showed that, for sufficient amplitudes $\left(\tau_{L} \gtrsim \tau_{\text {trap }}\right)$, the decay of an initial current modulation due to Landau damping can be strongly inhibited in the presence of space charge (nonlinear Landau damping). Therefore, linear Landau damping cannot serve as a possible saturation mechanism for the microwave instability in space charge dominated beams. Instead, space charge induced mode coupling phenomena are the dominant saturation mechanisms. In the longwavelength regime, wave steepening efficiently causes the decay of an initial current modulation. The corresponding damping rate is of the order of $\tau_{\text {break }}$. In the microwave region we find that the sudden merging of line density peaks is the dominant mode coupling mechanism that limits the growth of the current amplitudes in space charge dominated beams for $U_{\mathrm{sc}} / V_{\mathrm{bb}}=Z^{\mathrm{sc}} / Z^{\mathrm{bb}} \gtrsim 60$. Inside this boundary we still observe exponential growth, but the destructive effect of the microwave instability (phase space blowup) is strongly reduced. The long-time evolution is governed by the decay of the unstable mode into solitonlike structures. In addition to the persistent amount of coherent energy in solitonlike structures, kinetic energy is stored in a relatively small amount of decelerated ions forming a low velocity bump. A question that requires further work concerns the mechanism behind the observed long-time stability of the final distribution function.

[1] GSI Report No. 98-06, 1998, edited by I. Hofmann and G. Plass.

[2] K. Takayama, D. Arakawa, J. Kishiro, K. Koba, and M. Yoshii, Phys. Rev. Lett. 78, 871 (1997); T. Bohl, T. P. R. Linnecar, and E. Shaposhnikova, Phys. Rev. Lett. 78, 3109 (1997).

[3] J. J. Bisognano, in Proceedings of the Fifth European Particle Accelerator Conference, Barcelona 1996 (Institute of Physics, Bristol, UK, 1996), p. 328.

[4] G. Rumolo et al., Phys. Plasmas 6, 4349 (1999).

[5] O. Boine-Frankenheim, I. Hofmann, and G. Rumolo, Phys. Rev. Lett. 82, 3256 (1999).

[6] I. Hofmann, Laser Part. Beams 3, 1 (1985).

[7] G. Manfredi, Phys. Rev. Lett. 79, 2815 (1997).

[8] O. Boine-Frankenheim, Phys. Plasmas 3, 1585 (1996).

[9] S. G. Chen and G. Knorr, J. Comput. Phys. 22, 330 (1976).

[10] E. Fijalkow, Comput. Phys. Commun. 116, 319 (1999).

[11] Y. Chin and K. Yokoya, Phys. Rev. D 28, 2141 (1983); S. A. Bogacz and K. Y. Ng, Phys. Rev. D 36, 1538 (1987).

[12] R. C. Davidson, Methods in Nonlinear Plasma Theory (Academic, New York, 1972).

[13] P. L. Colestock, L. K. Spentzouris, and F. Ostiguy, in Proceedings of the 1995 Particle Accelerator Conference, Dallas (IEEE, Piscataway, NJ, 1996), p. 2757.

[14] I. B. Bernstein, J. M. Greene, and M. D. Kruskal, Phys. Rev. 108, 546 (1957).

[15] I. Hofmann, Fusion Eng. Des. 32-33, 33 (1996).

[16] S. I. Tzenov, in Workshop on Instabilities of High Intensity Hadron Beams in Rings, edited by T. Roser and S. Y. Zhang, AIP Conf. Proc. No. 496 (AIP, New York, 1999), p. 197. 